\title{
Narrative analysis to track the development of clinical reasoning during residency
}

\author{
Keshrie Naidoo*, Jane Baldwin, Jessie Lesar and Laura Plummer \\ Department of Physical Therapy, MGH Institute of Health Professions, Boston, MA, USA
}

Abstract

Purpose: Reflective narratives are valuable pedagogical tools that document and develop clinical reasoning. The purpose of this study was to investigate (through thematic analysis of reflective narratives) whether residents who were exposed to a specific clinical reasoning strategy showed development in the type and number of reasoning strategies used over the course of residency training.

Methods: This retrospective analysis used narrative research and thematic analysis of reflective narratives written by five residents over the course of a 12-month orthopaedic residency program. Narrative research involves the analysis of stories that are in text form. Residents were introduced into the clinical reasoning strategies (CRS) model, which describes eight types of reasoning strategies used by expert physical therapists: diagnostic, narrative, intervention procedures, interactive, collaborative, reasoning about teaching, predictive, and ethical reasoning. Researchers coded resident narratives for the types of CRS used by residents, and then analyzed the code frequency to describe the development of resident clinical reasoning.

Results: Researchers identified seven of the eight CRS strategies in resident narratives: diagnostic, narrative, reasoning about intervention procedures, interactive, collaborative, reasoning about teaching, and predictive reasoning. Residents used an average of 4.8 CRS at the beginning of residency and 5.8 at the end of the residency training. Reasoning about diagnostic and intervention procedures were used most frequently. Collaborative reasoning showed the most development, with $100 \%$ of the residents leveraging collaborative reasoning by the end of the residency.

Conclusion: Narratives serve as valuable pedagogical tools to help residents engage in reflective processes and understand the context of caring for their patients. This study illustrates using qualitative data analysis to evaluate the development of clinical reasoning skills throughout residency training. Clinical narratives can both foster and provide valuable insights into the development of clinical reasoning skills.

Keywords: clinical reasoning; reflective narratives; residency; constructivism; narrative research

Received: 20 June 2021; Revised: 2 I December 2021;Accepted: 31 December 2021; Published: I6 February 2022

$\mathrm{T}$ There are seven domains of competence for physical therapist (PT) residency education: clinical reasoning, knowledge for specialty practice, professionalism, communication, education, systems-based practice, and patient management. ${ }^{1}$ PTs who enroll in residency programs report valuing residency education for its influence on the development of clinical reasoning and professional skills. Residencies set their mission to develop the clinical reasoning of residents. ${ }^{2}$ However, few studies have investigated the influence of residency education on the development of clinical reasoning, which can affect patient outcomes. ${ }^{3,4}$ Additionally, educators lack consensus on what constitutes clinical reasoning and vary in their approaches to teaching and developing this skill, $, 5,6$ and there are few mechanisms to track the development of reasoning over the residency year., ${ }^{3,4,7}$

Clinical reasoning is described as 'the ability to organize, synthesize, integrate, and apply sound clinical rationale for patient management'. ${ }^{1}$ Currently, residency programs use live patient examinations (LPEs) and specialty-specific skills assessments to assess knowledge and clinical reasoning skills. ${ }^{1,8}$ When the LPE performance of graduating residents was compared with experience-matched PTs entering a residency program, those entering the residency scored $38.2 \%$ with a $0 \%$ pass rate, while the residency-trained PTs scored $83.4 \%$ with a $92.3 \%$ pass rate. $^{2}$ 
In a comparison of LPE scores among residents at the beginning and end of residency, examination and diagnosis showed a statistically significant change, while evaluation did not change significantly. ${ }^{3}$

While LPEs allow residents to showcase their clinical reasoning skills in action, narrative reflections allow residents to reflect on action and identify areas for development, such as clinical reasoning. Not only are narratives helpful for a resident's personal reflection but they also allow mentors access to residents' thought processes and allow for targeted feedback from mentors. Furthermore, narratives facilitate the development of moral agency and mindful practice - expert clinician attributes. ${ }^{9}$ Reflection requires active, persistent, and careful consideration. ${ }^{10} \mathrm{It}$ differs from rumination where experience is re-examined, but no action plans are developed. Reflection requires retrospective sense-making and prospective action clarification. Written reflections help to distance oneself from the event, decrease bias and the tendency to protect oneself, and help facilitate the development of detailed action plans. ${ }^{11}$

Narratives, which include a setting, characters, and a story arc, are currently used in PT residencies ${ }^{4,7}$ and medical residency programs ${ }^{12}$ to develop clinical reasoning, interpersonal skills, and ethical and reflective capacities. Pearson et al. concluded that the use of resident-authored narrative reflections during surgical residencies could measure competencies in areas such as communication, professionalism, systems-based practice, and practice-based learning. ${ }^{12}$ Narratives promote the humanistic aspect of patient care by uncovering aspects of the patient context, expanding clinical thinking, and facilitating a response to the contextualized healthcare environment. ${ }^{7}$

Expert PTs have been found to have fluid decision-making skills, which include both inductive and deductive reasoning and require the use of multiple types of reasoning strategies. ${ }^{13}$ Residents in this study were introduced into the clinical reasoning strategies (CRS) model described by Edwards et al. in 2004. ${ }^{13}$ The CRS model describes eight types of reasoning strategies used by expert PTs: diagnostic, narrative, intervention procedures, interactive, collaborative, reasoning about teaching, predictive, and ethical reasoning (see Table 1). The value of introducing the CRS model in residency education is that it makes visible the invisible aspect of clinical reasoning, ultimately making it easier for the resident to reflect on their reasoning and for mentors and faculty to provide feedback. Framed within the constructivist perspective, this study seeks to expand on the knowledge around the use of reflective narratives to understand the development of clinical reasoning in residency programs.

The framework that guides this study is the theory of constructivism, which equates learning with creating meaning from experience, and emphasizes that for learning to be meaningful and lasting, there must be practice in a contextualized setting. In the constructivist perspective, knowledge arises from the combination of action and reflection and the active manipulation of knowledge to create meaning. ${ }^{14}$ Learners create meaning rather than acquire it, emphasizing why constructivist instructional design techniques are most effective for advanced knowledge acquisition, ${ }^{14}$ such as during residency education. In this study, residents engaged in cognitive apprenticeship in the authentic environment, reflected on their practice verbally with mentors, wrote clinical narratives, and shared the narrative with fellow residents. The purpose of this study was to investigate through qualitative analysis of reflective narratives whether residents who were exposed to the CRS showed development in the type and number of reasoning strategies used during a 12-month orthopedic residency program.

\section{Methods}

\section{Design}

This retrospective analysis used narrative research and thematic analysis ${ }^{15,16}$ of narratives written by five residents. Residents wrote clinical narratives at four time points over the course of a 12-month residency program: 1 month, 5 months, 8 months, and 10 months, which were then subjected to analysis. Ethical approval was granted by the institution's Human Research Committee Institutional Review Board.

\section{Context and participants}

Narratives analyzed were written by a convenience sample of five residents who had graduated from one accredited orthopedic residency. In addition to patient care and educational hours, residents participated in community service, mentored scholarship, and served as laboratory instructors in a Doctor of Physical Therapy (DPT) program. Residents passed two LPEs and three written examinations, and met other graduation requirements to successfully complete the residency. The average age of the residents in this study was 25.8 years (range 24-29 years). Of the five residents, four were new graduates and one had 5 years of outpatient orthopedic experience.

\section{Clinical narratives and clinical reasoning strategies}

Residents selected a patient presenting with regionspecific complaints and submitted deidentified patient objective data, and followed this prompt 'Write a description of your clinical reasoning process, as best as you can recall, throughout your management of this patient, including the initial evaluation and 1-2 follow-up visits'. After submitting the first narrative, which served as a baseline, residents were introduced to the CRS model. ${ }^{13}$ Residents then reviewed a narrative written by an expert clinician to 
identify the types of CRS used. Residents also reflected on their first narrative, highlighting the types of CRS they would benefit from adding to their practice. Residents received feedback on their narratives from the residency director and facilitated a small-group discussion with colleagues around the patient interaction - engaging in the process of 'unbundling'?

\section{Analysis}

Narrative research is the analysis of stories of experience that are in text form. The narrative surrounds an event that is selected, organized, and presented to a particular audience. ${ }^{16}$ In this study, the stories of experience included a total of 20 resident narratives subjected to thematic analysis by the researchers. Three researchers established codes related to CRS a priori. These codes were labeled and operationally defined according to the reasoning procedure: diagnostic, narrative, intervention procedures, interactive, collaborative, reasoning about teaching, predictive, and ethical (Table 1). Researchers then independently coded an expert's narrative to achieve an intercoder agreement. Researchers reached an $85 \%$ agreement on coding the expert narrative, which is within the acceptable range of agreement. ${ }^{17}$ The reasoning strategies were found to be interconnected and overlapping, and some data excerpts needed to be coded as two types of reasoning strategies. Once excerpts were coded as more than one type of CRS, the researchers reached $100 \%$ agreement. The researchers then coded resident narratives independently and met to achieve intercoder agreement and determine the type and number of CRS used by residents. The focus of the thematic analysis was on 'what was said', that is, the type of reasoning strategy versus 'how it was said', that is, the quality of writing. ${ }^{16}$ The data were entered into NViVo qualitative software package ${ }^{18}$ to analyze the frequency of conceptual groups of the data. ${ }^{16}$

\section{Trustworthiness}

Researchers leveraged researcher triangulation and an audit trail to increase trustworthiness. ${ }^{19}$ Researcher triangulation included three researchers coding independently, and then meeting to achieve intercoder agreement. All research materials were kept in a central location, which produced an audit trail that allows for the study process to be replicated. The authors acknowledge their roles as research instruments during the data analysis process. The three researchers who completed coding $(\mathrm{KN}, \mathrm{JB}$, and LP) are PT educators with 12-20 years experience as educators in both DPT and residency education.

\section{Results}

Researchers analyzed a total of 20 narratives completed at four time points. Using the established coding strategy based on the CRS model, researchers identified seven of the eight CRS strategies in resident narratives. Evidence of ethical reasoning was not identified in any of the narratives. Residents used an average of $4.8 \mathrm{CRS}$ at the beginning and 5.8 near the end of the residency. Figure 1 highlights how, for instance, at Time 1 (T1, baseline reflective narrative) one out of the five residents used predictive reasoning but at Time 4 (T4, the final clinical reasoning assignment at the end of residency), four out of five residents were engaging in predictive reasoning with patients. Diagnostic and interventions procedures reasoning were used most frequently, whereas narrative reasoning was used infrequently. Collaborative reasoning showed the most development, with $100 \%$ of the residents leveraging it in their final reflective narrative.

\section{Diagnostic and intervention procedures reasoning}

Evidence of diagnostic and intervention procedures reasoning was found in $100 \%$ of the narratives analyzed. Residents moved through a systematic process to eliminate red flags and identify the need to refer or seek consultation:

I felt confident that her symptoms were mechanical, as the only red flags that she had were night pain and bilateral upper extremity involvement. Her night symptoms were related to rolling over in bed and resolved enough for her to fall asleep once she laid in the same position for a few minutes.

Having determined that a patient was appropriate for PT, residents then focused on making a physical therapy diagnosis. Multiple sources of information informed this reasoning process: patient and physician report, imaging, the resident's knowledge base and experience, as well as examinaton and re-examination data both within and between sessions.

\section{Predictive reasoning}

Predictive reasoning involves envisioning the future with the patient, providing information which informed whether to proceed with PT, and potential outcomes. Predictive reasoning was present in $25 \%$ of the first narratives and increased to $75 \%$ in the final narratives. Here, a resident weighs options that will impact the recovery time, 'I discussed the timeline of the disease process with the patient and informed her that sometimes in the early stage, patients benefit from a corticosteroid injection'.

As residents gained experience with patients with similar diagnoses, they were gaining insight into factors that can be either barriers or facilitators of recovery. Here, a resident considers the side effects of a medication and highlights how that knowledge informed her decision-making process: 
A recent note from her PCP showed that the patient reported increased irritability and mood swings after starting a prescription of Naproxen, which I have never heard before as a side effect of taking NSAIDs. Although I knew that these psychosocial implications were not the cause of symptoms, I wanted to be aware in order to account for presence of possible central sensitization, chronic pain behaviors, and possible barriers to prognosis.

\section{Reasoning about teaching}

Reasoning about teaching encompasses patient education and assessing whether intended learning occurred. Residents reflected on how they leveraged multiple modalities of education to ensure patient understanding. In this excerpt, a resident discusses their intentional decision-making to ensure safety with a home exercise program (HEP):

I attempt to build an initial HEP routine based around easier exercises that a patient can demonstrate good form and technique to ensure proper performance under unsupervised conditions. This allows us more time in-session to work on task-specific training and higher-level exercises that a patient may have difficulty repeating at home.

The next three forms of reasoning (narrative, collaborative, and interactive) were closely related with each form of reasoning informing one another.

\section{Narrative reasoning}

Narrative reasoning promotes understanding of the patient as a person, which is then integrated into reasoning and decision-making. While narrative reasoning strategies used trended down over the course of the residency year (Figure 1), in the following excerpt, a resident demonstrates an understanding of the patient's previous health experiences and how these may be influencing the patient's movement and comfort with activity:

I dug a little deeper with asking about activity level, when she revealed that ever since her radiation therapy, she had felt fatigued and had increased anxiety about being active. She seemed to think that being active would make the fatigue worse, and further the pain in her leg muscles. She stated she avoided activity for a very long time, which would additionally contribute to further deconditioning on top of the already occurring impairments in muscle strength, endurance, and aerobic capacity.

Leveraging narrative reasoning requires asking openended questions, thereby allowing the patient to share their illness or wellness story. One of the residents reflected on strategies for soliciting information rather than making assumptions:

As I learned about her history, I could understand why she seemed very anxious and frightened by the situation. I recognized that she had pressures on her from her children and work to get better quickly. Looking back, I now realize that I made an assumption about these feelings without validating whether they were true or not.

\section{Collaborative reasoning}

Collaborative reasoning incorporates valuing the patient's input and the inclusion of the patient as a source of knowledge, highlighting joint problem-solving and decision-making. Early in their training, one resident reflects on their lack of collaborative reasoning with the patient:

Looking back at these goals I notice that I didn't even take into consideration some of her activity and participation limitations. I should have made a goal surrounding her desire to get back into bowling/ fishing, being able to perform housework, transfers, ADLs.

At the end of residency, $100 \%$ of residents were using collaborative reasoning. Residents highlighted strategies for minimizing the power differential between a patient and therapist:

She was standing in the waiting room, sighing and shifting around. I brought her back to a treatment room and asked how she would be most comfortable while we talked about what was going on. I did this because I wanted her to know she had some choice in this situation and that my intent was to help her feel better, not worse. She chose to stand.

Even though it was challenging at times to relinquish control, residents reflected on the opportunity for growth in this area and ultimately valued this form of reasoning, 'It's definitely a challenge not to try and address everything at once, but I am trying to be better about focusing on what's most important to the patient to optimize function and participation goals'.

\section{Interactive reasoning}

Interactive reasoning also showed growth by the end of the residency, with $80 \%$ of residents using this strategy near the end. Interactive reasoning addresses how to approach and interact with patients to build rapport. Residents used interactive reasoning to think about additional 


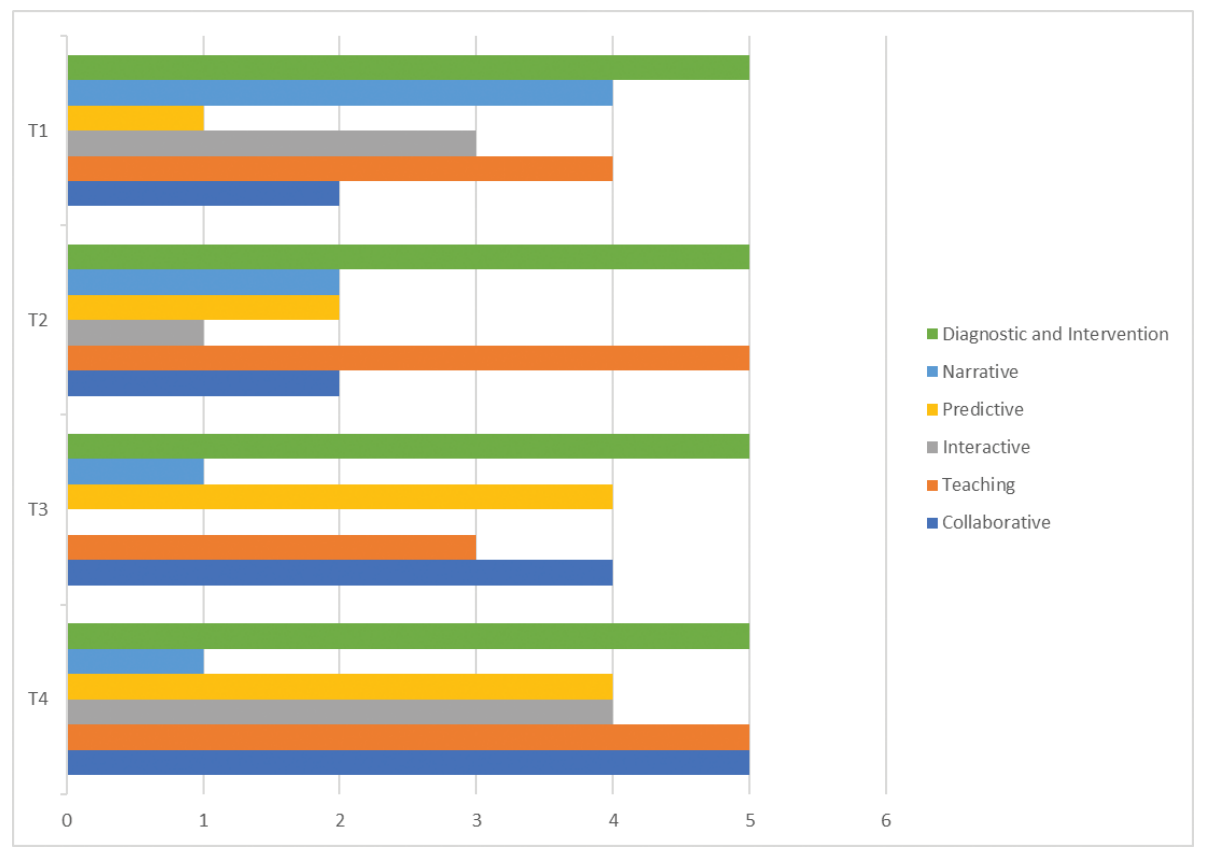

Fig. 1. Clinical reasoning strategies used by five residents at four time points.

Note: The $\mathrm{y}$-axis indicates time into the residency year: $\mathrm{T} 1=$ Time 1 (first clinical narrative written at 1 month), T2 = Time 2 (5 months), $\mathrm{T} 3=$ Time 3 ( 8 months), and T4 = Time 4 (10 months). The $\mathrm{x}$-axis indicates the number of residents who used the reasoning strategy.

intervention procedures they would need to leverage for a successful outcome:

At the same time, as a health care practitioner, I need to start also thinking, I wonder what stage of health behavior change they are in? How can I help them get to the next step? and, I wonder how fully previous health care providers have discussed this issue with the patient?

Residents would gather information about the success of their interactive strategies and make attempts to change their approach for the future. This form of reasoning helped residents make tough decisions about broaching harmful health behaviors with patients (see Table 1).

\section{Discussion}

This study explored the development of resident clinical reasoning after introduction to the CRS model through narrative research and thematic analysis of residents' written reflections. No other studies have analyzed PT resident narratives to explore the development of clinical reasoning, to date. Furze et al. explored the use of narratives to promote reflection during residency education and found that PT residents initially used singular reasoning and relied heavily on validation from mentors. Ultimately, residents transitioned to patient-focused collaborative reasoning and were better able to handle uncertainty in practice throughout residency. Residents also developed an improved pattern recognition, applying lessons learned from previous patients for improved care with current patients. ${ }^{4}$ Consistent with these findings, we found evidence of residents developing frameworks and tools to inform future practice and noted increased collaborative reasoning over the course of residency training. Additionally, an essential component of clinical reasoning is the ability to formulate hypotheses and evaluate a clinical problem effectively. ${ }^{6}$ Analysis of the narratives in this study revealed the evidence of diagnostic reasoning and reasoning about intervention procedure in $100 \%$ of resident narratives analyzed. It is unsurprising that this type of reasoning is on the forefront of residents' minds, given the focus on diagnosis and intervention during residency training.

Greenfield et al. highlight that narratives help clinicians make sense of their experience and the patient experience as well. ${ }^{7}$ We found the most growth in collaboration between a patient and provider, as $100 \%$ of residents were using collaborative reasoning by the end of the residency program. Additionally, as the reasoning strategies were overlapping and interconnected, we found that collaborative reasoning informed intervention procedures and reasoning about teaching, contributing to increased patient-centered care, a hallmark of expert practice. That reasoning is an iterative process became evident.

There are three types of knowledge used in PT practice: epistemic which refers to a discipline-specific knowledge base, phronetic knowledge, the procedural 
Table 1. Codebook including examples from expert and resident clinical narratives

\begin{tabular}{lll}
\hline Codes & Definition & Quotation \\
\hline Diagnostic reasoning & $\begin{array}{l}\text { Identifying impairments, activity lim- } \\
\text { itations and participation restrictions, } \\
\text { and other relevant contributing }\end{array}$ & $\begin{array}{l}\text { Predominant symptoms were headaches, mental fogginess, and dizziness. He reported a } \\
\text { factors }\end{array}$ \\
& $\begin{array}{l}\text { The patient reported that her pain was at her lateral hip, and it was painful all of the time, } \\
\text { especially at night. She indicated that rolling over in bed was difficult and the pain wakes her } \\
\text { up at night. She reported paresthesias down her leg, but these did not necessarily correspond } \\
\text { to her lateral hip pain. Knowing that she had a history of low back pain, I considered the pares- } \\
\text { thesias as separate symptoms from her lateral hip pain, until I could prove them to be related } \\
\text { (Resident 3, Narrative 4). }\end{array}$
\end{tabular}

Intervention proce- Decision-making around the dures reasoning

Predictive reasoning

Reasoning about teaching

Narrative reasoning

Understanding who the patient is, their context, culture, and their illness or wellness story

Collaborative reasoning

Attempts to minimize the powe differential between therapist and selection and administration of intervention and determining what to re-examine to inform determination of prognosis

Establishing a prognosis and influences decision making around the plan of care

Deciding on patient education strategies and assessing whether learning has occurred
As with all activities, physical exertion must be dosed carefully, with maximum heart rate monitored to control the level of exertion (Expert narrative)

I felt that strength and motor control were the two biggest areas to work on, specifically improving motor control of the $R$ quadriceps. During functional testing, she demonstrated decreased age-matched scores on the 5TSTS test and a knee extension lag with performance of the SLR. During the observation of gait, she demonstrated a Trendelenburg pattern indicative of hip abduction weakness. She also demonstrated knee hyperextension during gait and stair navigation, further, indicating quad weakness and loss of motor control. Although RL did have some knee ROM and mobility impairments, I did not think this was as high of a contributing factor as strength and motor control. (Resident 2, Narrative 4)

He frequently asked for my opinion as to whether he should return to school. I knew he wasn't ready but didn't want to dash his hope. I would simply detail the progress he had made thus far and re-state what we knew about his tolerance for activity at the present time (Expert narrative)

Given that she has increased symptoms later in the day as she wears a lead vest at work, it makes me think that she may require even longer to make the strength improvements necessary to maintain the endurance to wear the vest and maintain optimal lumbopelvic and LE posture and motor control for her work shifts, especially her $12-16 \mathrm{~h}$ shifts. (Resident 5, Narrative 4)

At this point, we had decreased his frequency to one time per month. [the patient] was now knowledgeable in his need to monitor and modify his activity level to maintain his symptom free state (Expert)

Interestingly, she avoided performing her HEP due to pain, but did switch back to two pillows, which helped to reduce her symptoms. I believe that she may have felt that performing her exercises may have aggravated her symptoms instead of addressing them. In retrospect, I wish I had spent more time educating her on the importance of continuing to move/ exercise within tolerance in order to address symptoms and to avoid extended bed rest. (Resident I, Narrative 3)

I could see in front of me a very defeated young man in the company of his very worried mother.They were looking to me for guidance. (Expert narrative)

She felt down and hopeless about not being able to fish or bowl like she used. She also was upset that she needed to ask for help from her family members because she was limited with being able to perform ADLs and household chores. She was used to being independent and felt ashamed that she now was relying quite often on others. Patient did use this as motivation though, expressing that she wanted to get better to be able to get back to the activities and no longer rely on others. (Resident 4, Narrative I)

By April he felt he had the tools to increase both cognitive and physical exertion levels independently, and we agreed that he would contact me as needed. (Expert narrative) patient/client and instead fostering a collaborative relationship
I took some time to educate her on the other approach, and that we would work together to find the best interventions for her. I was glad I had the stabilization route already cued up for her; I also realized that the patient would potentially benefit from a more self-managing and self-empowering intervention, when considering the patient from a chronic pain approach. (Resident 2, Narrative 2) 


\begin{tabular}{lll}
\hline Codes & Definition & Quotation \\
\hline Interactive reasoning & $\begin{array}{l}\text { The intentional choices the } \\
\text { therapist makes about how to } \\
\text { approach the patient/client and } \\
\text { establish rapport }\end{array}$ & $\begin{array}{l}\text { So I did what I always do and puffed myself up on the inside in order to exhibit just the right } \\
\text { level of confidence. I asked the right questions and listened attentively, nodding in an I under- } \\
\text { stand perfectly' kind of way. (Expert) }\end{array}$ \\
& $\begin{array}{l}\text { I have not broached the subject of smoking. She has seen many healthcare practitioners } \\
\text { and she has probably had discussions with these practitioners about smoking in the past. } \\
\text { It may be best to let her bring up this subject with me, rather than bringing it up myself, } \\
\text { Ethical reasoning }\end{array}$ & $\begin{array}{l}\text { because there may also be cultural influences on her smoking. I may approach this } \\
\text { subject at a later visit if she does not bring it up herself. (Resident 3, Narrative 4) } \\
\text { clinical practice taking the context } \\
\text { into consideration }\end{array}$ \\
\hline
\end{tabular}

knowledge integrating evidence-based practice, judgment and experience, and ethical knowledge. ${ }^{7}$ Despite ethical knowledge being vital to informing PT practice, we did not note any evidence of ethical reasoning in the resident narratives or the expert narrative we coded. This is likely due to the narrow operational definition used in this study rather than an absence of ethical reasoning across the residency year. Greenfield et al. highlight ethical knowledge and reasoning as guiding the patient towards decisions and engaging in decision-making in the best interests of patients and their stakeholders. ${ }^{7,20}$ An expanded view of ethical reasoning could have resulted in increased evidence in this study. We also noted that evidence of narrative reasoning decreased in frequency over the course of residency education. This may indicate that the CRS model should be revisited at multiple time points in the residency (as opposed to just the beginning) to incorporate principles of spaced practice into residency education. ${ }^{21,22}$

This study was grounded in constructivism and, within the situated learning model, cognitive apprenticeship, one of the hallmarks of residency education, is essential. ${ }^{23} \mathrm{Cog}$ nitive apprenticeship involves the learner completing tasks in the authentic environment, with the focus shifting to tasks that meet the needs of the learner. ${ }^{24}$ The emphasis is on the expert reasoning, which precedes and occurs during task completion. ${ }^{25}$ The development of clinical reasoning during residency education requires multiple elements: interaction with the patient in an authentic environment, debriefing with residency mentors, and written reflection. Collaborative inquiry with a small group of fellow residents can also facilitate reflection on action, thereby facilitating the knowledge construction process. ${ }^{7}$ This is consistent with the theory of constructivism, which highlights that for learning to be enduring, the learner must be presented with tasks and tools relevant to the setting, ${ }^{14}$ and there should be an interplay between activity, concept, and culture. ${ }^{23}$ This study focused on patient interaction and written reflection but missed the opportunity to analyze the rich discussion that takes place between a resident and a mentor, highlighting the need for further research.

\section{Limitations}

A limitation in this study includes thematic analysis conducted by a group of researchers from one institution with similar biases, which could influence the interpretation of the analysis. Additionally, while narratives can be found in written communication, narrative research traditionally includes interviews as well. ${ }^{16}$ This study is limited to written narratives guided by the reflection prompt provided to the residents. More reasoning strategies could emerge during debriefing sessions, which occur between residents and their mentor after a patient interaction. Future work would benefit from discourse analysis of the dialogue between residents and mentors. Additionally, one group of residents in one orthopedic residency program limits the transferability of findings. Future work would benefit from interviews with residents and mentors and the analysis of resident narratives from several institutions and across specialties.

\section{Conclusion}

Reflective narratives serve as valuable pedagogical tools to help residents engage in reflective processes and understand the context of caring for their patients. ${ }^{7}$ This study illustrated using qualitative data analysis to evaluate the development of clinical reasoning skills throughout residency education. Clinical narratives can both foster and provide valuable insights into the development of clinical reasoning skills.

\section{Disclosure}

Previous presentation of manuscript content: Poster presentation APTA Combined Sections Meeting 2021. 


\section{Ethical statement}

Ethical approval granted from the MGB Human Research Committee Institional Review Board (April 23, 2021, 2021P001015).

\section{Conflict of interest and funding}

The authors have no conflict of interest and funding to declare.

\section{References}

1. American Physical Therapy Association. Core competencies of a physical therapist resident January 2020. Available from: https://www.apta.org/contentassets/89db00a8ab01418c844ce d87e401563e/core-competencies-pt-resident.pdf [cited 20 June 2021].

2. Cunningham S, McFelea J. The influence of an orthopedic manual therapy residency program on improved knowledge, psychomotor skills and clinical reasoning in Nairobi, Kenya. Front Public Health (2017) 5: 1-5. doi: 10.3389/fpubh.2017.00055

3. Cunningham S, Litwin B, Fernandez-Fernandez A, et al. Influence of residency training on the clinical reasoning development of Kenyan physiotherapists. J Man Manip Ther (2019) 27(4): 237-44. doi: 10.1080/10669817.2019.1597434

4. Furze JA, Greenfield BH, Barr JB, et al. Clinical narratives in residency education: exploration of the learning process. Physiother Theory Pract (2019) 35: 1202-17. doi: 10.1080/09593985.2018.1472686

5. Gilliland S, Wainwright SF. Patterns of clinical reasoning in physical therapist students. Phys Ther (2017) 97(5): 499-511. doi: $10.1093 / \mathrm{ptj} / \mathrm{pzx} 028$

6. Wainwright SF, Gwyer J. (How) can we understand the development of clinical reasoning? J Phys Ther Educ (2017) 31(1): 4-6. doi: 10.1097/00001416-201731010-00003

7. Greenfield BH, Jensen GM, Delany CM, et al. Power and promise of narrative for advancing physical therapist education and practice. Phys Ther (2015) 95(6): 924-33. doi: 10.2522/ ptj.20140085

8. American Board of Physical Therapy Residency and Fellowship Education. Part III: quality Standards for Clinical Physical Therapist Residency and Fellowship Program June 2017. Available from: https://abptrfe.apta.org/globalassets/abptrfe/for-programs/abptrfe-part-iii-clinical-quality-standards.pdf [cited 20 June 2021].

9. Wainwright SF, Shepard KF, Harman LB, et al. Factors that influence the clinical decision making of novice and experienced physical therapists. Phys Ther (2011) 91(1): 91-101. doi: 10.2522/ ptj.20100161

10. Kolb DA. Experiential learning: experience as the source of learning and development. Englewood Cliffs, NJ: Prentice-Hall; 1984.

11. Nesbit PL. The role of self-reflection, emotional management of feedback, and self-regulation processes in self-directed leadership development. Hum Resour Dev Rev (2012) 11: 203-25.doi: $10.1177 / 1534484312439196$
12. Pearson A, McTigue M, Tarpley J. Narrative medicine in surgical education. J Surg Educ (2008) 65(2): 99-100. doi: 10.1016/j. jsurg.2007.11.008

13. Edwards I, Jones M, Carr J, et al. Clinical reasoning strategies in physical therapy. Phys Ther (2004) 84: 312-30. doi: 10.1093/ ptj/84.4.312

14. Ertmer PA, Newby TJ. Behaviorism, cognitivism, constructivism: comparing critical features from an instructional design perspective. Perform Improv Q (2013) 6: 50-72. doi: 10.1002/ piq. 21143

15. Lochmiller CR, Lester JN. An introduction to educational research: connecting methods to practice. Thousand Oaks, CA: Sage; 2017.

16. Riessman CK. Narrative analysis. Vol 30. Newbury Park, CA: Sage; 1993.

17. Miles MB, Huberman AM, Saldaña J. Chapter 12: writing about qualitative research. In: Miles MB, Huberman AM, Saldaña J, eds. Qualitative data analysis: a methods sourcebook. Thousand Oaks, CA: Sage; 2014, pp. 323-337.

18. QSR International Pty Ltd. (2020) NVivo (released in March 2020), Available from https://www.qsrinternational.com/ nvivo-qualitative-data-analysis-software/home

19. Creswell JW, Miller DL. Determining validity in qualitative inquiry. Theory Pract (2000) 39: 124-30. doi: 10.1207/ s15430421tip3903_2

20. Purtilo R, Doherty RA. Ethical dimensions in the health professions. 5th ed., St Louis, MO: Elsevier Saunders; 2013.

21. Karpicke JD, Roediger III HL. Expanding retrieval practice promotes short-term retention, but equally spaced retrieval enhances long-term retention. J Exper Psychol Learn Mem Cog (2007) 33(4): 704-19. doi: 10.1037/0278-7393.33.4.704

22. Roediger III HL, Pyc MA. Inexpensive techniques to improve education: applying cognitive psychology to enhance educational practice. JARMAC (2012) 1(4): 242-8. doi: 10.1016/j. jarmac.2012.09.002

23. Brown JS, Collins A, Duguid P. Situated cognition and the culture of learning. Educ Res (1989) 18: 32-42. doi: $10.3102 / 0013189$ X018001032

24. Lyons K, McLaughlin JE, Khanova J, et al. Cognitive apprenticeship in health sciences education: a qualitative review. Adv Health Sci Educ. (2017) 22(3): 723-39. doi: 10.1007/ s10459-016-9707-4

25. Woolley NN, Jarvis Y. Situated cognition and cognitive apprenticeship: a model for teaching and learning clinical skills in a technologically rich and authentic learning environment. Nurse Educ Today (2007) 27(1): 73-9. doi: 10.1016/j. nedt.2006.02.010

\footnotetext{
* Keshrie Naidoo

Department of Physical Therapy

MGH Institute of Health Professions,

36 First Avenue, Charlestown Navy Yard

Boston, MA 02129, USA

Email:knaidoo@mghihp.edu
} 